\title{
Management versus Mangel
}

\author{
Personalmanagement ist mehr als Disposition vorhandenen Personals - Planen, \\ ge-winnen, motivieren, binden - das und vieles mehr gehört zum Personalmanagement. Wie \\ aber Personalmanagement betreiben, wenn der Fachkräftemangel weiter fortschreitet, Fach- \\ personal nicht mehr ausreichend oder gar nicht zur Verfügung steht?
}

$\boldsymbol{\nabla}$ Es ist unbestritten, dass die Angebotsvielfalt ambulanter Dienste und anderer Dienstleister im sozialen und pflegerischen Bereich zunehmen wird. Dabei wird es nicht nur darum gehen, medizinische und pflegerische Leistungen mit Fachkräften zu erbringen. Auch bei niederschwelligen Dienstleistungen werden die Kunden immer höhere Qualität erwarten. Über den Erfolg eines Dienstleisters entscheiden also die Qualifikation der Mitarbeiter und die Qualität ihrer Arbeit. Auf Grund des Nachwuchsmangels und der Fluktuation wird es allerdings künftig sowohl im pflegerischen als auch in ergänzenden Bereichen schwieriger werden, Mitarbeiter zu gewinnen, die motiviert und fachlich versiert sind, um Dienstleistungen an und um den Konsumenten Mensch/Patient zu erbringen.

\section{Doppelstrategie verfolgen}

Für einen Dienstleister gibt es nur einen Weg: Er muss seinen Betrieb so attraktiv machen, dass er eine starke Anziehungskraft auf Arbeitnehmer ausübt. Fachkräfte $\mathrm{zu}$ gewinnen wird in Zukunft nicht ausschließlich davon abhängen, welche Stundenlöhne gezahlt werden.

Potenzielle Mitarbeiter werden deutlich mehr auf das Betriebsklima, den Ruf der Einrichtung sowie die individuellen Verwirklichungschancen im Betrieb angepasst an die Lebensplanung - achten. Dabei spielen natürlich auch die Rahmenbedingungen und besondere Leistungen von Arbeitgebern eine Rolle. In absehbarer Zeit wird gleichermaßen um
Kunden/Patienten und um versiertes Personal gerungen werden. Dem wird nur mit einer doppelten Strategie am Markt $\mathrm{zu}$ Leibe $\mathrm{zu}$ rücken sein.

Aufgabe des Personalmanagement ist also auch die Gestaltung und Vermarktung des Leistungsanbieters mit all seinen Fassetten, Chancen und Risiken. Ideal ist es, wenn es dem Personalmanagement gelingt, dass die einmal gewonnenen Mitarbeiter ihr Unternehmen mitformen, mitgestalten, voll dahinter stehen und es nach außen transparent präsentieren. Je besser dies in Zukunft umgesetzt wird, desto leichter wird ein Unternehmen Fachpersonal auf einem fast leergefegten Markt bekommen und binden können. Für wenig innovative Unternehmen könnte es hier zu Engpässen bis hin zur Aufgabe der Marktteilhabe führen.

Auch der MDK trägt durch die Veröffentlichungen der MDK-Prüfberichte in dieser Sache Verantwortung. Die Berichte, wie sie in der jetzigen Form gestaltet sind, verzerren das Bild. Und die eigentlich "guten Betriebe“ werden weder von Kunden noch von potenziellen Arbeitnehmern identifiziert. Hier muss dringend nachgebessert werden.

\section{Das brauchen wir}

Wir brauchen in Deutschland mehr gut ausgebildete Pflegende und Mitarbeiter in ergänzenden Dienstleitungen. Generalistische Pflegeausbildung, deutliche Erhöhung der Ausbildungsplätze und eine bessere finanzielle Ausstattung der Einrichtungen sind die Voraussetzungen, um mehr junge Menschen für den Pfle- geberuf zu begeistern. Dazu gehört auch, dass es eine neue und klare Definition pflegerischen Handelns in Abgrenzung $\mathrm{zu}$ anderen Professionen geben muss. In diesem Kontext sind Stichpunkte wie Berufsgesetz, Pflegekammer, Vorbehaltsaufgaben zu nennen.

Spannend bleibt die Frage, wie sich der Konsument verhalten wird in Bezug auf die Bereitschaft, Einrichtungen und damit bestimmte Mitarbeiter zu wählen und den entsprechenden Preis für qualitativ hochwertige Leistungen zu bezahlen. Gerade in der ambulanten Pflege besteht jetzt noch einmal die Chance, aus den immer noch vorhandenen Klischees - wie Helfen, Dienen und Gutes tun herauszukommen, das Image des Pflegeberufes aufzubessern. Es geht darum, das eigene Handeln innovativ zu beschreiben und damit das Produkt "Pflege“ als Dienstleistung am Markt zu platzieren.

- Thomas Meißner

- Vorstand - Anbieterverband qualitätsorientierter Gesundheitspflegeeinrichtungen e.V.; Geschäftsführer Häusliche Pflege Meißner \& Walter GmbH Alt Biesdorf 71a, 12683 Berlin

- Email: buero@meissner-walter.de 